\title{
Gamificación en el aula de matemáticas
}

\author{
María Dolores Yélamos Moya \\ Universidad Almería, Almería, loly1801@hotmail.com: \\ Presentado en eXIDO18 (2018)

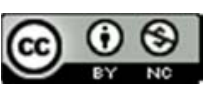

\section{RESUMEN}

En este trabajo se estudiará la técnica de gamificación aplicada a la educación, donde se pretende modificar el comportamiento del alumnado mediante las técnicas propias de los juegos. El trabajo se dividirá en las siguientes partes:

- Una introducción del concepto de gamificación.

- Propuesta del sistema gamificado, donde aplicaremos las herramientas de la gamificación a la enseñanza de matemáticas en $2^{\circ}$ ESO con el tema de "Áreas y volúmenes" respetando la metodología y el sistema de evaluación que ya estaban fijados en la programación de la asignatura. Para el diseño de este sistema gamificado, se han considerado los gustos del alumnado y se ha cogido como base la serie de dibujos animados Dragon Ball y diferentes personajes que están dentro de sus intereses, hobbies, etc.

En este sistema gamificado se ha diseñado: contexto del juego, avatares, niveles, medallas, recompensas, retos o misiones y puntos, clasificaciones y el cálculo de la nota en función del número de puntos que consigan.

- Explicación de la parte del sistema gamificado llevado a cabo durante el periodo de prácticas. Esta parte consta de un concurso, donde los alumnos/as tendrán que competir por equipos para ganar. Finalmente se dará a conocer algunas de las opiniones del alumnado.

- Conclusiones obtenidas.

Palabras clave: gamificación, motivación, sistema gamificado y juego.

\section{INTRODUCCIÓN}

Nunca os habéis preguntado, ¿cómo puedo conseguir que una o varias personas cambien su comportamiento para realizar una acción, que a veces, no es muy deseada? Por ejemplo, una persona perezosa ir al gimnasio o que nuestro alumnado quiera aprender más. La respuesta es muy sencilla, 
simplemente es cuestión de motivar. ¿Y cómo podemos motivar? Una buena solución es jugando.

Antiguamente había otro tipo de motivaciones pero la sociedad ha ido evolucionando a un ritmo rápido, nos encontramos en una generación donde la información rebosa, rodeada de tecnología y videojuegos, y acostumbrada a recibir muchos estímulos exteriores. La sociedad avanza rápidamente y nosotros tenemos que hacerlo con ella, adaptándonos a la era digital.

Por todo esto surgió el concepto de gamificación donde se usan los elementos propios de los juegos, en contextos no lúdicos, para modificar el comportamiento de los individuos mediante su motivación.

Hoy en día es un problema la falta de motivación que se encuentra en las aulas y por ello el docente debería de trabajarla, para poder transmitir los conocimientos de forma eficaz. Como posible solución a este problema haremos uso de la gamificación. Para ello se ha diseñado un sistema gamificado para el alumnado de $2^{\circ} \mathrm{ESO}$ en matemáticas y se particularizará para incluir la experiencia llevada a cabo durante el periodo de prácticas con el Tema de Área y volúmenes. Todo este sistema se ha diseñado respetando la metodología y el sistema de evaluación que ya estaban fijados en la programación de la asignatura. No se pudo llevar a cabo todo lo que se recoge en este trabajo, pero se tuvo la oportunidad de poner en práctica la parte del concurso que explicaremos más adelante.

\section{METODOLOGÍA}

A la primera pregunta que tendremos que responder es ¿por qué se quiere usar la gamificación? Nuestro gran objetivo es que el alumnado esté motivado, que aprenda y que tenga un buen rendimiento académico, para ello es necesario que realice sus tareas, que tenga en la libreta todos los apuntes dados para que posteriormente dispongan de toda la información necesaria para poder estudiar y que participen más, es decir, que se impliquen más. 
Área temática

Para la evaluación del trabajo del alumnado se tendrá en cuenta que la programación de la asignatura especifica los siguientes apartados:

1. Examen: cuya nota será un $70 \%$ de la calificación total. Se realizará el último día de clase.

2. Proyecto: donde se trabajará en grupos cooperativos de cuatro y cinco integrantes. Representará el $10 \%$ de la nota final y el modo de llevarlo a cabo será mediante un concurso cuya ejecución serán los dos días previos al examen.

3. Libretas: donde deberán tener los apuntes dictados en clase y todos los ejercicios hechos correctamente. Será el $10 \%$ de la nota final. Se recogerán el día del examen para su corrección y se devolverán el siguiente día.

4. Trabajo diario: donde se puntuará el salir a pizarra, hacer la tarea y contestar a preguntas realizadas en clase. Se llevará un seguimiento diario en un cuaderno.

Para el diseño de este sistema gamificado, se ha considerado los gustos del alumnado y se ha cogido como base la serie de dibujos animados Dragon Ball y diferentes personajes que están dentro de sus intereses, hobbies, etc.

\section{Contexto del juego}

Inicialmente se les pondrá en contexto con la siguiente narración, donde incorporaremos imágenes de algunos personajes de la serie Dragon Ball. 
Una vez más Goku está en problemas, erróneamente pidió al Shenlon Rojo (dragón de las siete bolas) convertirse en niño. Tras ello las siete bolas de dragón se dispersan por diferentes lugares, y para conseguirlas habrá que superar diferentes retos. Si no se recolectan en menos de un año, la Tierra desaparecerá. Para evitarlo, iremos a por ellas, pero Goku es consciente de que no puede hacerlo solo y nos pide que lo acompañemos en su viaje, así que juntos lucharemos para conseguirlo. ¡A por ello!

\section{Avatares}

El alumnado elegirá su propio avatar, el cual lo identificará en esta aventura.

Estos corresponderán a personajes famosos de su agrado.

Para la selección de estos avatares se ha tenido en cuenta sus gustos y que son 33 alumnos/as, por ello se ha elegido 60 posibles opciones para que tengan un amplio abanico de elección. Dato a tener en cuenta, no se pueden repetir.

\section{Niveles}

Al principio del juego, cada jugador partirá con 100 puntos y estará en el nivel 'Goku', conforme vaya ganando puntos de las diferentes actividades irá subiendo de nivel. Para conseguir ayudar a Goku y obtener las siete bolas de dragón habrá que llegar al nivel 'Super Saiyan Blue'.

Los niveles existentes y las respectivas puntuaciones para estar en ellos son los siguientes: 
Área temática

Tabla 1. Niveles.

Nivel

Puntos

$<750$ puntos

Goku

750 puntos - 24999 puntos

Super Saiyan 1 
Área temática

25000 puntos -36499 puntos

Super Saiyan 2

Super Saiyan 3
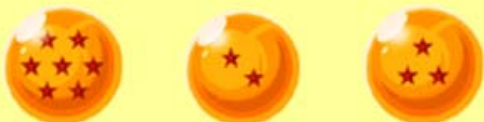

36500 puntos -53999 puntos
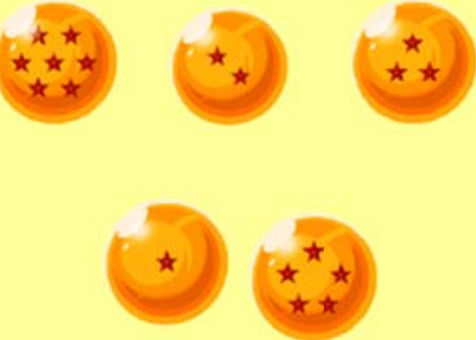

54000 puntos -72999 puntos

Super Saiyan 4

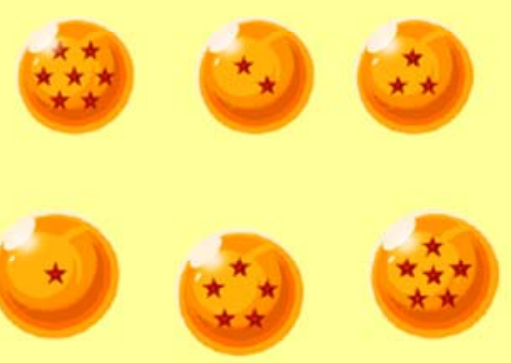

$\geq 73000$ puntos

Super Saiyan Blue

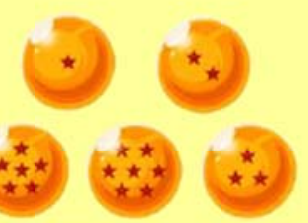


Área temática

\section{Medallas}

Al pasar de nivel se dará una insignia, que será una bola de canica y que representará una bola de dragón (todos los días al finalizar la clase se tendrán que devolver y se volverán a entregar al principio de la siguiente clase).

\section{Recompensas}

Al recibir una bola de dragón se obtendrá una recompensa. Esta recompensa será una tarjeta que elegirá entre las que les dé el profesor/a. Cada tarjeta les otorga un poder, que podrán usar cuando crean oportuno (es decir, no tienen que usarla nada más ganarla), y muy importante, solo se puede usar una vez. Una vez usada, el jugador ya no dispondrá de ella.

Las recompensas al alcanzar determinados niveles son los siguientes:

Nivel 'Super Saiyan 1'. Se disponen de tres tipos de cartas, que son las siguientes:
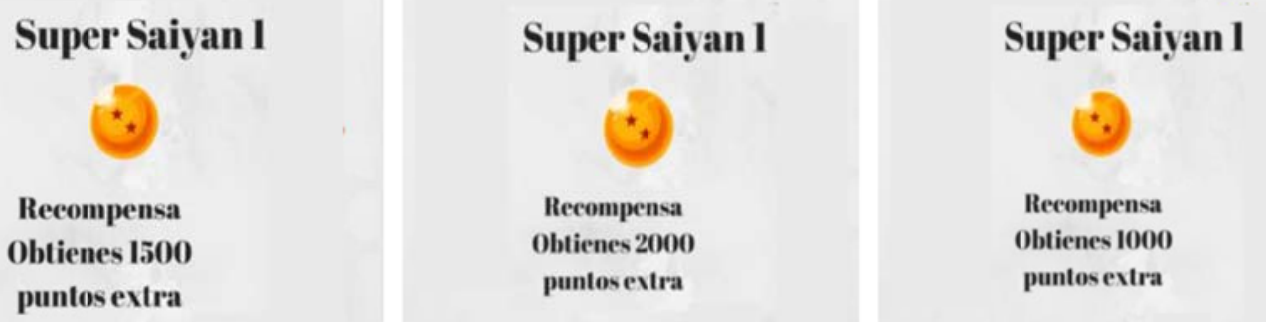

Nivel 'Super Saiyan 2': Se disponen de dos tipos de cartas, que son las siguientes: 
Área temática

\section{Super Saiyan 2}

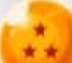

Recompensa

Multiplicas por 2 los

puntos obtenidos por

una acción

\section{Super Saiyan 2}

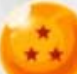

Recompensa

Vultiplieas por el número que te salga en el dado los puntos obtenidos por una acción

Nivel 'Super Saiyan 3': Se disponen de tres tipos de cartas, que son las siguientes:
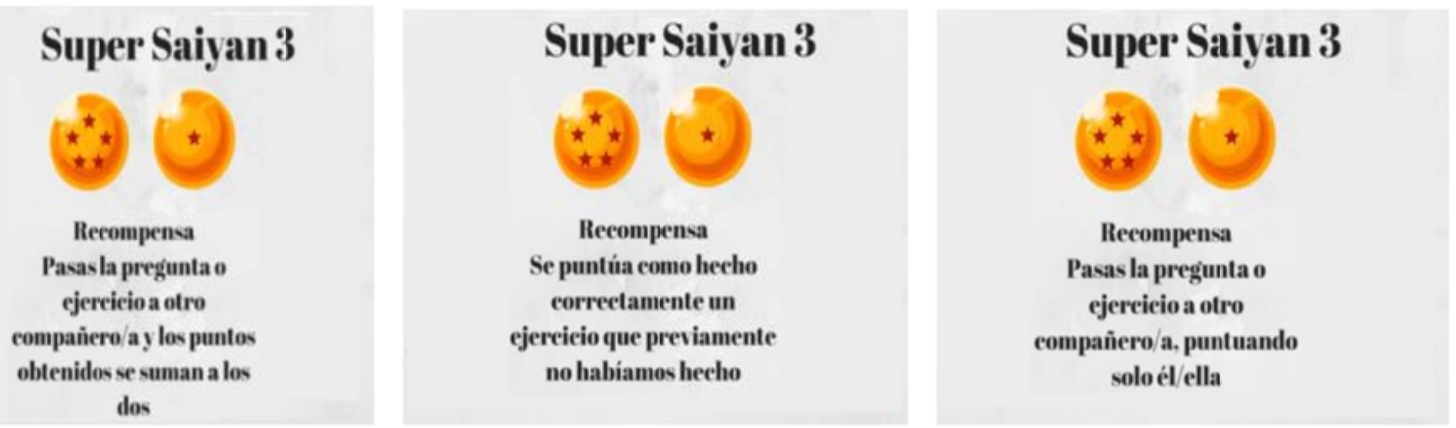

Nivel 'Super Saiyan 4': Se disponen de dos tipos de cartas, que son las siguientes:

\section{Super Saiyan 4}

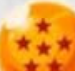

Recompensa

Puedes pedir una pista de

cómo resolver un ejercicio

en el concurso

\section{Super Saiyan 4}

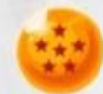

Recompensa

En el caso de fallar una pregiunta del concurso podemos elegir a que grupo va como pregunta rebote

Nivel 'Super Saiyan Blue': Solo se tendría un tipo de carta y es la siguiente: 
Área temática

\section{Super Saiyan Blue}

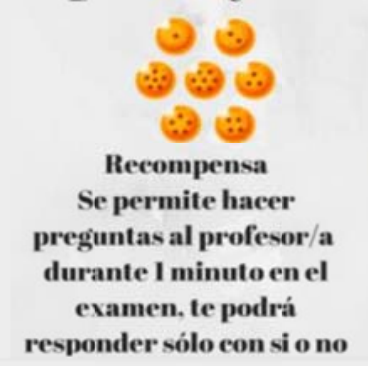

La forma de elección de la carta en cada uno de los niveles es a suerte.

Pero también tendremos recompensas inesperadas:

1. Al pasar del nivel 'Super Saiyan 2' al nivel 'Super Saiyan 3', no recibirán una bola de dragón, sino dos.

2. Cuando todos lleguen a un nivel en concreto, se tendrán dos recompensas colectivas:

2.1. Cuando alcancen el nivel 'Super Saiyan 1' se les dirá una posible pregunta que les puede tocar en el primer día del concurso.

2.2. Cuando alcancen el nivel 'Super Saiyan 3' se les dirá un posible problema a resolver que les puede tocar en el segundo día del concurso.

\section{Segunda parte de la historia}

El día previo al concurso se pasará a la segunda parte de la historia, junto al texto podremos incorporar imágenes de la serie Dragon Ball: 


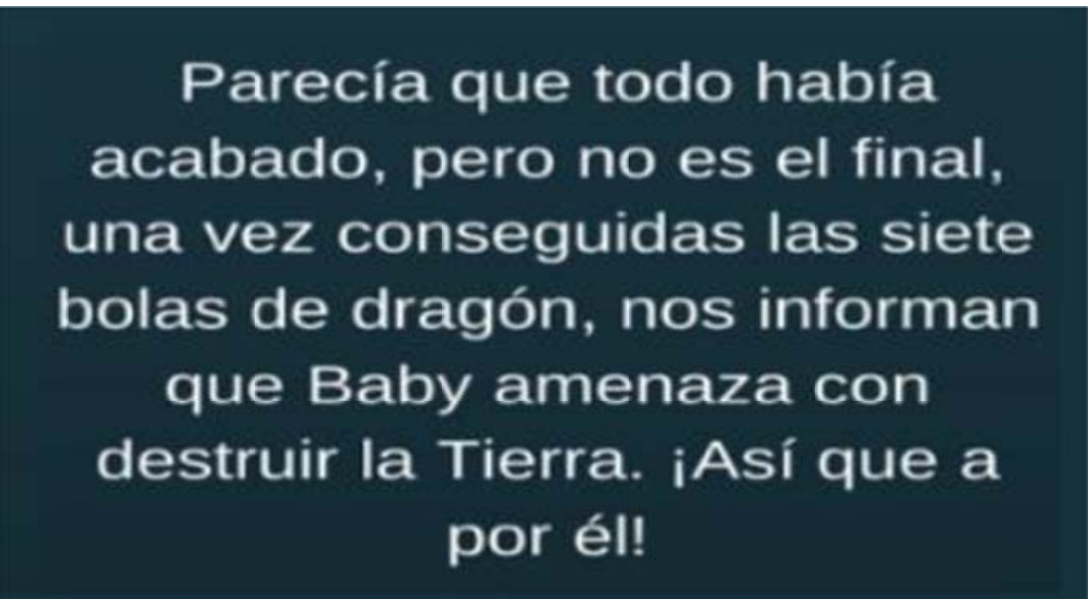

Una vez se haya alcanzado el nivel 'Super Saiyan Blue' y reunidas las siete bolas de dragón, dependiendo de la puntuación podemos obtener distintos finales del juego:

Tabla 2. Diferentes finales del juego.

\begin{tabular}{|c|c|c|}
\hline Nivel & Puntos & Final \\
\hline \multirow{3}{*}{ Super Saiyan Blue } & 151421 puntos - 194683 puntos & $\begin{array}{l}\text { No consigues } \\
\text { derrotar a Baby y la } \\
\text { Tierra desaparece }\end{array}$ \\
\hline & 194684 puntos - 216314 puntos & $\begin{array}{l}\text { Después de mucho } \\
\text { sufrimiento derrotas a } \\
\text { Baby }\end{array}$ \\
\hline & $\geq 216315$ puntos & $\begin{array}{l}\text { Derrotas a Baby } \\
\text { fácilmente con la } \\
\text { ayuda de Pan }\end{array}$ \\
\hline
\end{tabular}

\section{Descripción del concurso que forma parte del proyecto}

Se ha diseñado este sistema gamificado en base al alumnado de $2^{\circ}$ ESO, tomando en consideración sus gustos, hobbies, número de ejercicios, preguntas de exámenes, recursos disponibles, limitaciones,... En lo que respecta a la experiencia durante el periodo de prácticas se ha podido llevar a 
Área temática

cabo una parte de este sistema gamificado, el concurso, que procederemos a explicar en qué consistió exactamente.

Primeramente se dividió la clase de 33 alumnos/as en siete grupos, cinco grupos con cinco componentes y dos grupos de cuatro componentes. Los equipos no tenían ningún nombre especial en esta experiencia, pero dentro del contexto que estamos describiendo se pedirá a los alumnos/as que elijan el nombre de su equipo.

Esta actividad se desarrolló en dos días y para cada día se diseñó un tipo de tarjeta diferente, pero siempre con el mismo patrón:

- Parte delantera (la que veía el alumnado): el dibujo de un sólido platónico (era bastante interesante por el tema tratado y porque se había hecho referencia a ellos en clases anteriores).

- Parte trasera (la que no veía el alumnado): donde se formulaba la pregunta o problema a resolver, el tiempo del que iban disponer para resolverla y su solución, para poder verificarla rápidamente.

\section{Tarjetas del primer día.}

Un ejemplo es:
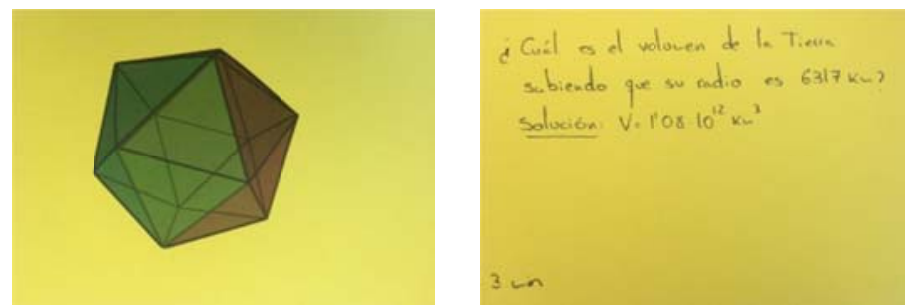
Área temática

\section{Tarjetas del segundo día.}

Un ejemplo es:
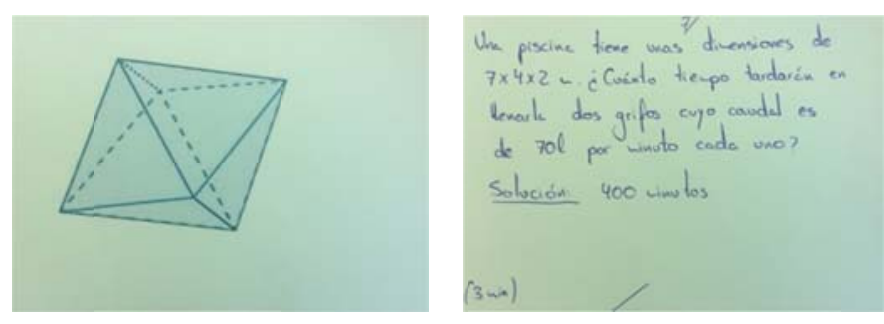

Existen diferencias entre las tarjetas del primer y segundo día, y no es solo el color. La diferencia radica en la dificultad de las preguntas, en las del Día 1, el nivel de dificultad es bajo/medio y se piden cálculos de áreas (en 2 minutos) y volúmenes (en 3 minutos), en cambio las del Día 2 tienen un nivel de dificultad medio/alto y piden la resolución de problemas de áreas (en 3 minutos) y de volúmenes (en 4 minutos).

A la hora de realizar el juego en clase, se distribuyeron por equipos, se les adjudicó un número de equipo y cada equipo eligió su representante, cuyas funciones eran dar la respuesta consensuada por el equipo y elegir la tarjeta a resolver en su turno. Todos tenían que estar orientados hacia la pizarra, lugar donde aparecería la clasificación general, con el nombre de cadla equipo y sus puntuaciones respectivas. Se apuntaban además los puntos que se le anotarían, en caso de acertar, en función del tiempo que se les daba para resolver la cuestión y cuánto tardaban en resolverlo. 
Área temática

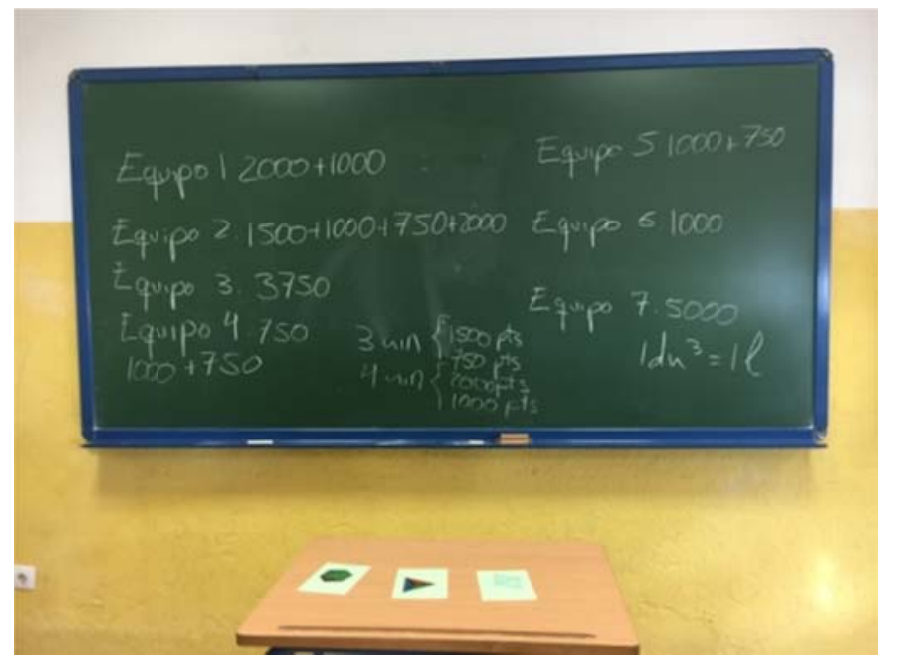

Nos situábamos delante de ellos con una mesa, donde estaban los diferentes montones de tarjetas (cada montón estaba compuesto por las tarjetas con las mismas representaciones de los sólidos platónicos), habiendo el primer día cuatro montones y el segundo día tres.

Las reglas se comentaron rápidamente y fueron fáciles de entender:

- Solo podía dar respuesta el representante del equipo seleccionado.

- Se respetarían los tiempos dados, los turnos de palabra y las decisiones tomadas.

El incumplimiento de estas reglas, se consideraría incumplimiento de normas de clase, por lo que se restarían 500 puntos al equipo procedente de quien o quienes realizaban dichos incumplimientos.

El tiempo se midió con pulsómetro y se iba avisando del tiempo restante cada minuto.

La mecánica del juego era sencilla:

Empezaba un grupo al azar, se dijo un número cualquiera y se contó los grupos en sentido contrario a las agujas del reloj, y en ese mismo sentido, se hizo el orden de turnos. 
El representante del equipo (en su turno) debía levantarse a elegir uno de los montones de cartas, se situaba y se procedía a formular la pregunta en voz alta para todos los grupos.

Cuando un equipo tenía el turno, solo el representante de ese equipo podía contestar a la pregunta en el tiempo marcado, si acertaba puntuaba y si no, podía seguir intentándolo hasta agotar su tiempo. Una vez acabado el tiempo, si el grupo no acertaba, dicha pregunta pasaba a ser pregunta rebote y el siguiente equipo podría contestarla, si acertaba puntuaba, si no pasaba como pregunta rebote y así sucesivamente. En el caso de que ningún equipo supiese la respuesta, dicha pregunta quedaba invalidada (situación que no se dio).

Para evitar que solo trabajase el equipo que tenía el turno, se evaluó el concurso de tal forma que, por un lado contarían las puntuaciones obtenidas por cada equipo y por otro lado, se evaluaría un folio/s que debían de entregar al final del concurso y donde tendrían que estar contestadas correctamente todas las preguntas realizadas durante el concurso, independientemente de a qué equipo fuesen dirigidas dichas preguntas. Es obvio, que antes de empezar el concurso se les informó de cómo se les iba a evaluar. En el apartado retos y puntos que aparece más adelante se detalla la forma de puntuar cada pregunta.

El último día del concurso, 10 minutos antes de acabar la clase se procedió a la entrega de premios. Se suponía que el grupo que obtuviese más puntos sería el único que obtuviese recompensa (recompensa aleatoria), pero se llamó a cada uno de los representantes de los equipo no ganadores y se sacó sus recompensas (inesperadas) de una caja, la recompensa era una bolsa de chuches para cada equipo. Se consideró lo más justo, puesto que todos los equipos se mostraron participativos y se esforzaron. La recompensa (aleatoria) para el equipo ganador fue también una bolsa de chuches para el equipo, huesitos y un tablerone. La elección del tablerone fue porque en clases anteriores se había puesto como ejemplo de un prisma triangular y esto les llamó mucho la atención. 
Área temática

\section{Retos o misiones y puntos}

Los diferentes retos y misiones están basados en el sistema de evaluación anteriormente descrito y que incluía un examen, proyecto, libreta y trabajo diario. La superación de cada una de las misiones llevará consigo unas puntuaciones, con un máximo de 209000 puntos, que servirán para calcular la nota correspondiente a cada apartado y que ya está establecida en la programación de la asignatura.

\section{EXAMEN}

El examen constará de cuatro ejercicios, con diferentes apartados cada uno.

Tabla 3. Examen.

\begin{tabular}{|c|c|c|c|}
\hline \multicolumn{3}{|c|}{ Sumas puntos si } & Puntos \\
\hline \multicolumn{3}{|c|}{+} & + \\
\hline \multirow{3}{*}{ Ejercicio 1} & Apartado a) & Correcto & 1125 puntos \\
\hline & Apartado b) & Correcto & 1125 puntos \\
\hline & Apartado c) & Correcto & 2250 puntos \\
\hline \multirow[t]{2}{*}{ Ejercicio 2} & Apartado a) & Correcto & 1500 puntos \\
\hline & Apartado b) & Correcto & 1500 puntos \\
\hline \multirow{3}{*}{ Ejercicio 3} & Apartado a) & Correcto & 1500 puntos \\
\hline & Apartado b) & Correcto & 1500 puntos \\
\hline & Apartado c) & Correcto & 1500 puntos \\
\hline Ejercicio 4 & & Correcto & 3000 puntos \\
\hline $\begin{array}{l}\text { Ejercicios 1, 2, } 3 \\
\text { y } 4\end{array}$ & & $\begin{array}{l}\text { Si están a } \\
\text { medias }\end{array}$ & $\begin{array}{c}\text { Puntuación proporcional } \\
\text { correspondiente }\end{array}$ \\
\hline
\end{tabular}


Área temática

\section{PROYECTO (Concurso)}

Se tendrá en cuenta para la asignación de puntos el tiempo en minutos, $\mathrm{t}$, en el que han realizado la tarea. La tarea se desarrolla en dos días.

Tabla 4. Proyecto.

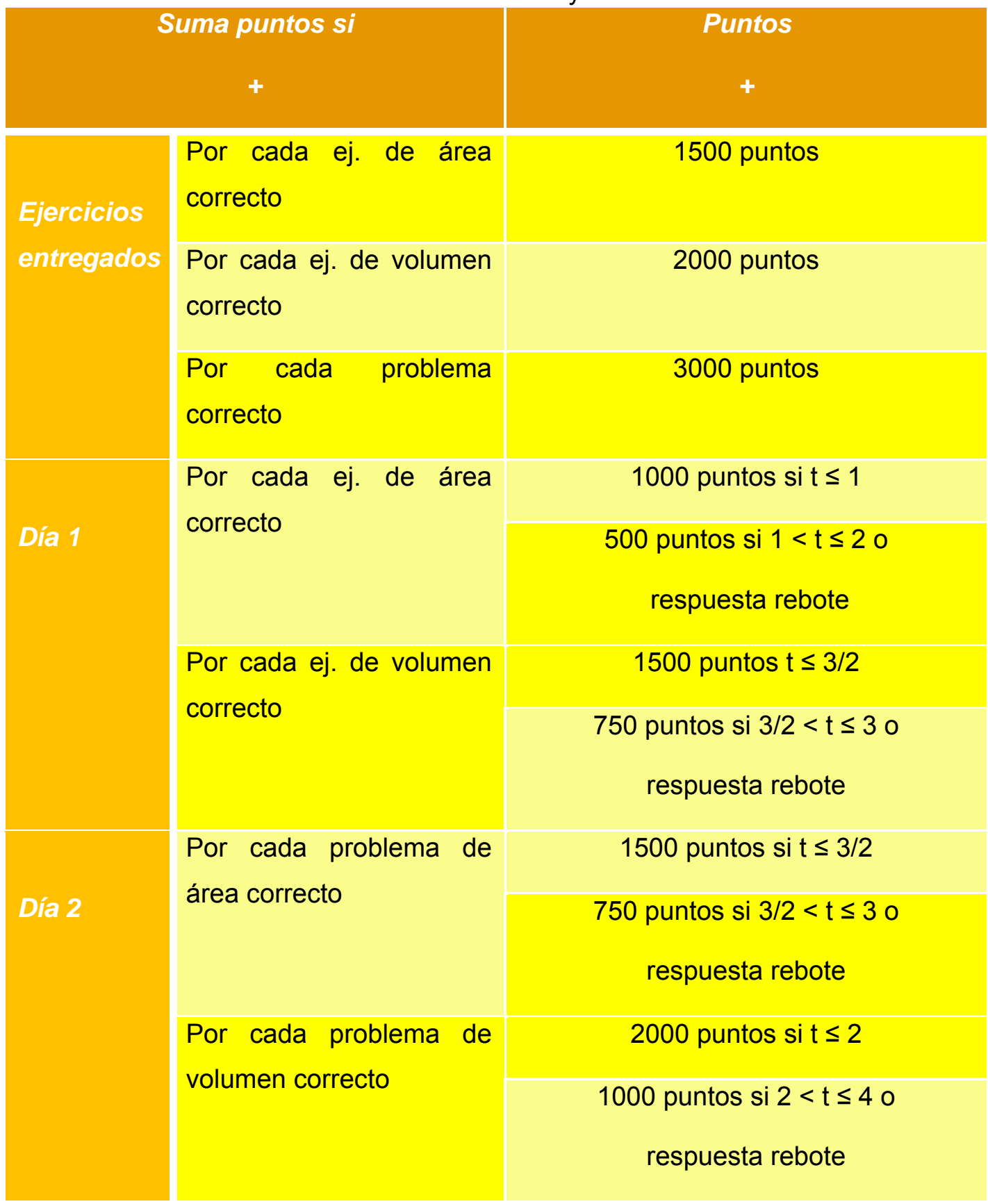


Área temática

3. LIBRETA

Tabla 5. Libreta.

\begin{tabular}{|c|c|}
\hline \multicolumn{1}{c|}{$\begin{array}{c}\text { Sumas puntos si } \\
+\end{array}$} & Puntos \\
\hline Por cada ej. de área correcto & + \\
\hline Por cada ej. de volumen correcto & 1500 puntos \\
\hline Por cada apuntes dictados & 2000 puntos \\
\hline
\end{tabular}

4. TRABAJO DIARIO

Tabla 6. Trabajo diario.

\begin{tabular}{l|l}
\multicolumn{1}{|c|}{$\begin{array}{c}\text { Sumas puntos si } \\
+\end{array}$} & \multicolumn{1}{c}{$\begin{array}{c}\text { Puntos } \\
+\end{array}$} \\
\hline Por cada ejercicio hecho correctamente de \\
tarea
\end{tabular}

COMPORTAMIENTO

Tabla 7. Comportamiento

\begin{tabular}{|cc|}
\hline Resta puntos si & Puntos \\
No respeta las normas de clase & - \\
\hline
\end{tabular}




\section{Clasificaciones}

Los puntos que vayan consiguiendo cada uno de los jugadores se irán apuntando. Cada día, al llegar a clase se mostrará en pizarra la clasificación general. Esta misma clasificación la podrán ver la tarde anterior en la red social Instagram (cuenta cerrada que solo podrán acceder los jugadores y cuya finalidad es informar).

\section{Cálculo de la nota}

La nota final sobre 10 que obtendrá el alumnado se calculará a partir de los puntos obtenidos en el examen, proyecto, libreta y trabajo diario teniendo en cuenta los pesos fijados en la programación de la asignatura. En la siguiente tabla se puede ver la correspondencia entre puntos y nota máxima en cada apartado.

Tabla 8. Puntos y notas

\begin{tabular}{|l|c|c|}
\hline & $\begin{array}{c}\text { Número máximo de } \\
\text { puntos que se pueden } \\
\text { conseguir }\end{array}$ & Nota \\
\hline Examen & 15500 puntos & 7 \\
\hline Proyecto & 50500 puntos & 1 \\
\hline Libreta & 70000 puntos & 1 \\
\hline Trabajo diario & 73000 puntos & 1 \\
\hline
\end{tabular}

\section{RESULTADOS}

El feedback del alumnado durante el concurso fue euforia, alegría, pique y diversión. Pero, ¿cómo se podía saber sin basarse meramente en la observación? Al finalizar la entrega de premios, se les pidió que en una hoja, de forma anónima, describieran qué les había parecido la experiencia y que la 
Área temática

introdujeran en la misma caja donde anteriormente se había llevado los premios (la elección de la caja fue para que no pudiesen ver las recompensas).

Algunos de los comentarios escritos por el alumnado son los siguientes:
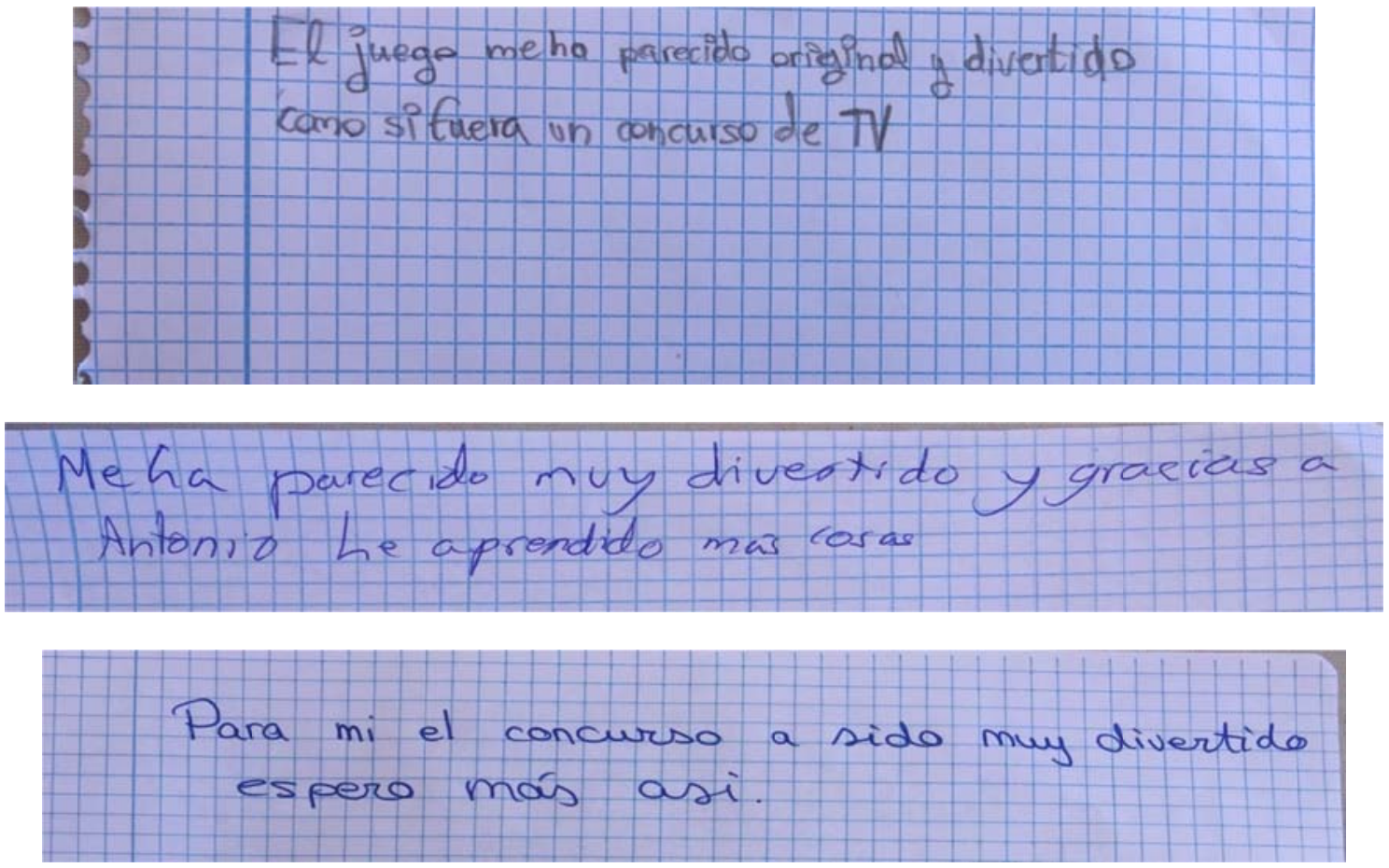

¿Qué técnicas de gamificación usamos en el concurso?

Según la descripción del concurso se puede decir que estuvieron presentes:

Mecánicas: Puntos, clasificaciones (por equipo en pizarra) y retos o misiones (cada pregunta realizada).

Dinámicas: Recompensas aleatorias (para el equipo ganador), recompensas inesperadas (para el resto de equipos), competición, estatus, logro, altruismo, feedback y diversión.

Estéticas: Reto.

\section{CONCLUSIONES}

- La gamificación puede ser una buena herramienta para aumentar la motivación.

- Se tiene que conocer a los jugadores y diseñar el juego exclusivamente para ellos. 
Área temática

- Se adapta a las nuevas generaciones.

- Se puede llevar a la práctica de forma exitosa.

- Se requiere mucho tiempo y esfuerzo para su diseño y desarrollo.

\section{REFERENCIAS}

Bartle, R. (2003). Designing Virtual Worlds. New Riders.

Carpena, N., Cataldi, M. y Muñiz, G. (2012). En busca de nuevas metodologías y herramientas aplicables a la educación. Repensando nuestro rol docente en las aulas. Argentina. Recuperado el 12/6/2018 de: http://papers.cumincad.org/data/works/att/sigradi2012 85.content.pdf

Csikszentmihalyi, M. (1990). Flow: The Psichology of Optimal Experience. New York: Harper \& Row.

Dale, E. (1969). Audio-visual methods in teaching. New York: Dryden.

Hunicke, R., LeBlanc, M. y Zubeck, R. (2004). MDA: A formal approach to game design and game research. Recuperado el 12/6/2018 de: http://www.cs.northwestern.edu/ hunicke/MDA.pdf

Kapp, K. M. (2012). The gamification of learning and instruction: game-based methods and strategies for training and education. San Francisco: Pfeiffer.

Kumar, J. y Herger, M. (2013). Gamification at Work: Designing Engaging Business Software.

McGonigal, J. (2012). Reality is broken. Londres: Vintage Books.

Ramírez Cogollor, J. L. (2014). Gamificación. Mecánicas de juegos en tu vida personal y profesional. Madrid: SClibro(Grupo RC).

Sutton-Smith, B. (2001). Ambiguity of Play. Harvard Business Review.

Teixes, F. (2015). Gamificación. Motivar jugando. Barcelona: UOC. 
Área temática

Werbach, K. y Hunter, D. (2014). Revoluciona tu negocio con las técnicas de los juegos. Madrid: Pearson.

Yee, N. (2007). Motivations of Play in Online Games. Journal of CyberPsichology 9, 772-775.

Zicherman, G. (2011). The purpose of gamification. A look at gamification's applications and limitations. Recuperado el 12/6/2018 de:

http://radar.oreilly.com/2011/04/gamification-purpose-marketing.html 